\title{
The heart-brain team: neurocardiology
}

\section{El equipo corazón-cerebro: neurocardiología}

\author{
Amado Jiménez-Ruiz" ${ }^{*}$, José L. Ruiz-Sandoval², Diego Araiza-Garaygordobiß ${ }^{3}$, and Daniel Sierra-Lara ${ }^{3}$ \\ ${ }^{1}$ Stroke, Dementia \& Heart Disease Lab, Western University, Ontario, Canada; ${ }^{2}$ Neurology Department, Hospital Civil Fray Antonio Alcalde, \\ Guadalajara, Jalisco, Mexico; ${ }^{3}$ Cardiology Department, Instituto Nacional de Cardiología Ignacio Chávez, Mexico City, Mexico
}

Cardiovascular and cerebrovascular diseases are among the main causes of morbidity and mortality in Mexico and the world. The nervous system and the cardiovascular system share an intrinsic network of physiological and pathological mechanisms that are important for both the neurologist and the cardiologist. Neurocardiology covers heart disorders that affect the brain, brain disorders that affect the heart and systemic diseases that affect both organs. Cerebrovascular disease is the most important cause of disability, the second leading cause of cognitive impairment and the main cause of epilepsy in older adults.

Herein, we present some examples that help to understand the possible implications between both systems (Fig. 1).

\section{Cardioembolic stroke}

This mechanism corresponds to $25 \%$ of all strokes, especially in the context of atrial fibrillation (AF), thus generating high morbidity and mortality, mainly in the geriatric population. AF-related strokes tend to be more severe and disabling than those produced by other mechanisms ${ }^{1,2}$. Two concepts that question what we thought we knew have been recently described: the first one is that the presence of $A F$ is not necessary for intracavitary thrombosis

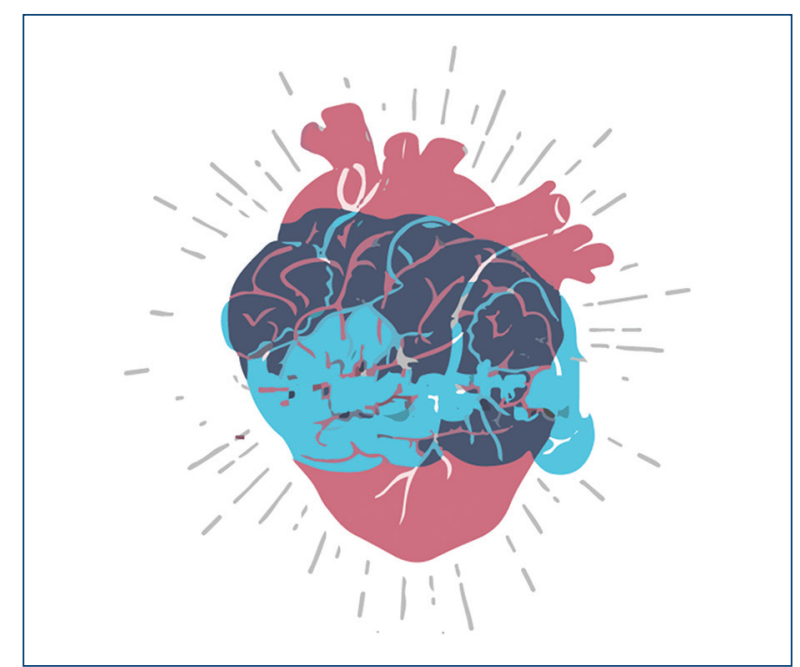

Figure 1. Heart-brain.

to be generated; and the second, that AF can have a cardiac, cerebral, or mixed origin. The left atrium undergoes electrical, structural and functional changes that can initiate thrombogenesis, even in the absence of demonstrable arrhythmia. This process is known as atrial cardiopathy and opens a new line of research in the field of myocardial dysfunction inflammatory and immune factors, even in the absence of demonstrable arrhythmias ${ }^{3}$.

\section{Correspondence:}

*Amado Jiménez-Ruiz

E-mail: dr.amadojimenez@gmail.com
Date of reception: 11-05-2020

Date of acceptance: 06-10-2020 DOI: 10.24875/ACME.M21000234
Available online: $20-10-2021$ Arch Cardiol Mex (Eng). 2021;91(3):357-359 www.archivoscardiologia.com 2604-7063 / @ 2020 Instituto Nacional de Cardiología Ignacio Chávez. Published by Permanyer. This is an open access article under the CC BY-NC-ND license (http://creativecommons.org/licenses/by-nc-nd/4.0/). 
Insular strokes are proof that autonomic nervous system control influences on cardiac modulation control, causing heart rate alterations and arrhythmias. In these cases, atrial fibrillation (AF) could be a direct cause of brain damage, i.e., neurogenic, while that which is detected prior to the stroke could be a direct cause of myocardial damage, i.e., cardiogenic. Making the distinction could influence on natural evolution of the disease and its prognosis 4 .

\section{Systemic diseases}

Autoimmune diseases, such as systemic lupus erythematosus, often occur with endothelial dysfunction expressed as vasculitis in different organs (including the heart and brain), and constitute one of the main causes of morbidity and mortality in this population ${ }^{5,6}$.

Mitochondrial diseases are another example of systemic diseases that cause cardiac and neurological alterations, with the common denominator of cellular energy system failure ${ }^{7,8}$.

The main causes of sudden cardiac arrest are ischemic heart disease, cardiomyopathies and primary arrhythmic disease ${ }^{9}$.

Breakthroughs in advanced cardiac support prehospital protocols have increased the number of cardiac arrest survivors in intensive care units, and the latest guidelines include recommendations for mechanical ventilation, sedation and induced hypothermia within the first 6 hours after the event ${ }^{10}$.

\section{Cerebral amyloid angiopathy, atrial fibrillation and cognitive impairment}

With population aging, finding geriatric patients with $\mathrm{AF}$ and cerebral amyloid angiopathy is increasingly common. The latter is caused by $\beta$-amyloid build-up in cerebral vessels and parenchyma. Clinical presentation consists of cognitive impairment and recurrent intracerebral hemorrhage, especially in patients taking oral anticoagulants. Despite this association, it is not included within current diagnostic algorithms and risk scales. Determining cerebral amyloid angiopathy presence and lesion burden prior to initiating oral anticoagulation for AF could be an important step to prevent intracerebral hemorrhage ${ }^{11}$.

Mutations in the genes responsible for familial Alzheimer's disease (PSEN 1, PSEN 2 and APP) and subsequent $\beta$-amyloid protein build-up could explain part of the link between cardiac aging, heart failure development and neurodegenerative diseases occurrence as a multisystemic disease model ${ }^{12,13}$. There is broad observational evidence that associates AF itself with cognitive impairment, both of the vascular type and Alzheimer's disease. Possible mechanisms include clinical or silent stroke, intracerebral hemorrhage, global hypoperfusion and the increased $\beta$-amyloid deposition in the brain ${ }^{14}$. Anticoagulation might reduce the risk of cognitive impairment in these patients ${ }^{15}$.

Cerebrovascular disease coexists in most neurodegenerative diseases that involve cognitive impairment and, currently, it is the only treatable and potentially preventable component of these disorders ${ }^{16}$.

\section{Hypertension}

Systemic hypertension is the most important modifiable cardiovascular risk factor for both the cardiologist and the neurologist. Hypertensive intracerebral hemorrhage is the leading cause of intracerebral hemorrhage in Mexico and the world. Hypertension is associated with small vessel disease, including white matter disease, lacunar stroke, microhemorrhages and cognitive impairment. The SPRINT MIND study demonstrated (by neuropsychological follow-up and brain imaging studies) that blood pressure intensive control decreases the risk of mild cognitive impairment, and extending this follow-up to a long term might decrease the risk of major neurocognitive disorder (formerly dementia) ${ }^{17}$.

\section{Dyslipidemia}

Dyslipidemia, especially as regards low-density lipoprotein, is a cornerstone of cardiovascular events primary and secondary prevention. There is evidence that very low levels could be associated with intracerebral hemorrhage, especially in women ${ }^{18}$, and this risk factor is not stratified within current diagnostic and therapeutic algorithms either. Nevertheless, a meta-analysis concludes that the benefits of lipid reduction therapy in the prevention of stroke largely outweigh the risk of intracerebral hemorrhage, and should not deter doctors from treating this metabolic disorder ${ }^{19}$.

\section{Subarachnoid hemorrhage}

Most patients with aneurysmal subarachnoid hemorrhage exhibit electrocardiographic alterations, as well as troponin elevation. Sudden death, myocardial stunning 
and stress-induced cardiomyopathy (Takotsubo syndrome) are attributed to a catecholamine storm, owing to damage to the nervous system.

\section{Other examples}

- Embolic protection in procedures such as transcatheter aortic valve implantation (TAVI) and other brain complications associated with cardiac procedures.

- Implantable devices for occult atrial fibrillation detection after stroke.

- Stroke, paradoxical embolism, migraine and patent foramen ovale.

- Biomarkers in cardio-cerebral disease diagnosis (troponins, natriuretic peptide, D-dimer).

- Prognostic factors for hypoxic-ischemic encephalopathy after cardiac arrest and brain death diagnosis.

- Heart rhythm disorders diagnosis and treatment in patients with systemic diseases (e.g., muscular dystrophy and genetic-origin ataxia)

\section{Conclusion}

There are many links between neurology and cardiology. It is necessary for both types of professionals to be integrated in heart-brain teams that participate in the prevention, diagnosis and treatment of those diseases that cause more morbidity and mortality in our country ${ }^{20}$.

\section{Acknowledgements}

We thank Almendra Covarrubias for the creation of the image on figure 1.

\section{Funding}

This research has not received any specific grant from agencies of the public, commercial, or non-profit sectors.

\section{Conflicts of interest}

None.

\section{Ethical disclosures}

Protection of human and animal subjects. The authors declare that no experiments have been performed on humans or animals for this investigation.

Confidentiality of data. The authors declare that they have followed the protocols of their work center on the publication of patient data.

Right to privacy and informed consent. The authors declare that no patient data appear in this article.

\section{References}

1. Griñán $\mathrm{K}$, Arboix A, Massons J, Díez L, Vergés E, Gil F, et al. Cardioembolic stroke: risk factors, clinical features, and early outcome in 956 consecutive patients. Rev Invest Clin. 2020 May 7;73(5). doi: 10.24875/ RIC.20000227. Online ahead of print.

2. Campbell BCV, Khatri P. Stroke. Lancet. 2020;396:129-42.

3. Leifer D, Rundek T. Atrial cardiopathy: a new cause for stroke? Neurology. 2019;92:155-6.

4. Cerasuolo JO, Cipriano LE, Sposato LA. The complexity of atrial fibrillation newly diagnosed after ischemic stroke and transient ischemic attack: advances and uncertainties. Curr Opin Neurol. 2017;30:28-37.

5. Mavrogeni S, Koutsogeorgopoulou L, Dimitroulas T, Markousis-Mavrogenis G, Boki K, Katsifis G, et al. Combined brain/heart magnetic resonance imaging in systemic lupus erythematosus. Curr Cardiol Rev. 2020;16:178-86.

6. Calle-Botero E, Abril A. Lupus vasculitis. Curr Rheumatol Rep. 2020;22:71.

7. Behjati M, Sabri MR, Etemadi Far M, Nejati M. Cardiac complications in inherited mitochondrial diseases. Heart Fail Rev. 2020 Jul 29. doi: 10.1007/s10741-020-10009-1. Online ahead of print.

8. Lim A, Thomas RH. The mitochondrial epilepsies. Eur J Paediatr Neurol 2020;24:47-52.

9. Rodríguez-Reyes $\mathrm{H}$, Muñoz-Gutiérrez $\mathrm{M}$, Salas-Pacheco JL. Current behavior of sudden cardiac arrest and sudden death. Arch Cardiol Mex. 2020;90:183-9.

10. Cronberg T, Greer DM, Lilia G, Moulaert V, Swindell P, Rossetti AO. Brain injury after cardiac arrest: from prognostication of comatose patients to rehabilitation. Lancet Neurol. 2020;19:611-22.

11. DeSimone CV, Graff-Radford J, El-Harasis MA, Rabinstein AA, Asirvatham SJ, Holmes DR. Cerebral amyloid angiopathy. J Am Coll Cardiol. 2017:70:1173-82.

12. Cortes-Canteli M, ladecola $C$. Alzheimer's disease and vascular aging: JACC Focus Seminar. J Am Coll Cardiol. 2020;75:942-51.

13. Yang M, Li C, Zhang Y, Ren J. Interrelationship between Alzheimer's disease and cardiac dysfunction: the brain-heart continuum? Acta Biochim Biophys Sin. 2020:52:1-8.

14. Sepehri Shamloo A, Dagres N, Müssigbrodt A, Stauber A, Kircher S, Richter S, et al. Atrial fibrillation and cognitive impairment: new insights and future directions. Heart Lung Circ. 2020;29:69-85.

15. Diener H-C, Hart RG, Koudstaal PJ, Lane DA, Lip GYH. Atrial fibrillation and cognitive function: JACC Review Topic of the Week. J Am Coll Cardiol. 2019;73:612-9.

16. Hachinski V. Dementia: paradigm shifting into high gear. Alzheimers Dement. 2019:15:985-94

17. Zamora Z, Williamson JD. The effects of lower BP goals on cognitive function in the elderly. Curr Cardiol Rep. 2020;22:63.

18. Rist PM, Buring JE, Ridker PM, Kase CS, Kurth T, Rexrode KM. Lipid levels and the risk of hemorrhagic stroke among women. Neurology. 2019;92:e2286-94.

19. Judge C, Ruttledge S, Costello M, Murphy R, Loughlin E, Álvarez-Iglesias A, et al. Lipid Lowering Therapy, Low-Density Lipoprotein Level and Risk of intracerebral hemorrhage - a meta-analysis. J Stroke Cerebrovasc Dis. 2019:28:1703-9.

20. Alkhouli M, Graff-Radford J, Holmes DR. The heart-brain team - towards optimal team-based coordinated care. JAMA Cardiol. 2018;3:187. 